\title{
Effect of Prophylactic llioinguinal Neurectomy on Postoperative Groin Pain Following Lichenstein Hernioplasty
}

\author{
Atif Sharif1, Tasleem Akhtar1, Mumtaz Akhtar2, Irfan Malik3, Muhammad Hanif4 and Naeem Zia4
}

\begin{abstract}
Objective: To compare mean postoperative pain post-Lichenstein open hernioplasty with and without ilioinguinal neurectomy at six months.

Study Design: Randomised controlled trail.

Place and Duration of Study: Surgical Unit-I, Benazir Bhutto Hospital, Rawalpindi, from August 2014 to February 2015. Methodology: Adult male patients with unilateral reducible inguinal hernia, who consented to the study between the age range of 18-80 years, were included. Recurrent, irreducible or strangulated, or large inguinal-scrotal hernia and those with previous abdominal incision, impaired cognition, peripheral neuropathy, limited mobility and females were excluded. Patients were equally randomised to nerve-preservation and excision groups. Mann-Whitney U-test was applied to find out difference in inguinodynia at 1 and 6 months.

Results: There was significant difference in pain at 1 month in the nerve-preservation group ( $M d=6.00, I Q R=4, n=90)$ and nerve excision group $(M d=3.50, I Q R=4, n=90), U=2308.00, z=-5.017, p<.001$ and at 6 months in the nerve preservation group ( $M d=2.00, I Q R=1, n=90$ ) and nerve-excision group ( $M d=0.00, I Q R=1, n=90), U=3001.00, z=-3.470, p=0.001$.

Conclusion: Prophylactic ilioinguinal neurectomy significantly reduces groin pain at 6 month as compared to nerve preservation group following Lichenstein hernioplasty.
\end{abstract}

Key Words: Lichenstien hernioplasty, Chronic groin pain, llioinguinal neurectomy, Visual analog score.

\section{INTRODUCTION}

Lichenstein mesh inguinal hernioplasty is one of the commonest surgeries performed worldwide.1,2 Operative management of an inguinal hernia is successful in majority with minimum postoperative sequelae, a few weeks convalescence, ${ }^{2}$ and no long-term complications. Inguinodynia or chronic groin pain following the procedure, however, has emerged as a potential complication. Incidence of such debilitating chronic groin pain, which restricted patient's daily activities and affected employment ranged from $0.5 \%$ to $6 \%$ following hernia repairs. 4 Chronic groin pain following hernia repair remains vastly under-reported, 4,5 and only $1 \%$ of such patients are referred for further treatment of pain. 4

There is a symptom complex of neuropathic and nonneuropathic pain. The neuropathic causes include inadvertent entrapment of nerve in suture or mesh, perineural fibrosis, partial division, crushing of nerve, or diathermy burns, 2,3 triggered by hyperextension of the hip and walking. The non-neuropathic pain is actually

1 Department of Vascular Surgery, Russells Hall Hospital, Dudley, England

2 Surgical Unit, Al Nafees Medical College, Islamabad, Pakistan

3 Surgical Unit, The District Headquarters (DHQ) Hospital, Rawalpindi, Pakistan

4 Surgical Unit-I, Benazir Bhutto Hospital, Rawalpindi, Pakistan

Correspondence: Dr. Tasleem Akhtar, Department of Vascular

Surgery, Russells Hall Hospital, Dudley, England

E-mail:tasleem_1@hotmail.com

Received: May 19, 2017; Accepted: January 18, 2019 somatic pain due to periosteal reaction, scar tissue, mechanical pressure caused by folded mesh called 'meshoma' and visceral pain, which is experienced during ejaculation. Neuropathic causes, i.e. chronic nerve irritation etc, are deemed the main cause of postoperative inguinodynia. ${ }^{6}$

The ilioinguinal nerve runs in the operative field, i.e immediately below the cut external oblique fascia and may get entangled in hernia repair and reapproximation of external oblique fascia. ${ }^{6}$ Non-identification of the ilioinguinal nerve leading to inadvertent damage to nerve, during open inguinal hernioplasty, is the major risk for this chronic groin pain. ${ }^{7}$

Multiple studies have been done on prophylactic neurectomies with good results. But until recently, chronic inguinodynia is a problem with no definition, known etiology or classification, and definitive surgical treatment. Thorough groin neuroanatomy knowledge is pivitol, as to prevent this complication, the best way being prevention of nerve injuries. ${ }^{8}$

In the reference study by Khoshmohabat et al., mean inguinal pain at one month after surgery was $0.56 \pm 0.5$ in nerve resection group compared to $1.89 \pm 0.6$ in nerve preservation group $(p<0.001) .{ }^{2}$ Similar statistical results were observed at 6 and 12 months follow-up, showing ilioinguinal nerve resection at the time of inguinal hernioplasty, decreasing postoperative groin pain, and it can be made a routine practice in inguinal hernioplasty.

There are different views available in the professional literature regarding prophylactic ilioinguinal neurectomy; 
and no consensus has been developed so far. This study might help in establishing a safe and practical method for prevention of postoperative chronic groin pain following Lichenstein open hernioplasty.

The objective of this study was to compare the postoperative pain in patients undergoing Lichenstein mesh hernioplasty with and without ilioinguinal neurectomy at 6 months.

\section{METHODOLOGY}

It was carried out as a randomised controlled trail at the Surgical Unit-I, Benazir Bhutto Hospital, Rawalpindi, from August 2014 to February 2015. The sample was selected by consecutive (non-probability) sampling technique. Included were male patients of age between 18 and 80 years having unilateral hernia and undergoing Lichenstein open hernioplasty under spinal anesthesia. Bilateral, recurrent, irreducible or strangulated hernia, large inguinal-scrotal hernia, impaired cognition, peripheral neuropathy, limited mobility patients, and females were excluded.

After Ethical Committee approval and informed consent, patients were randomly assigned to either neurectomy [Group A] or control group [Group B] using the lottery method. In study group, the ilioinguinal nerve was cut 3 to $4 \mathrm{~cm}$, sharply with a blade. The proximal end was embedded into the internal oblique without tension after transection with no drain. A polypropylene mesh was placed as per standard technique.

To minimise loss to follow up, full contact details, including the contact number of the participants, were noted down at the start of the study and they were reminded of the follow-up appointment at 30th and 180th postoperative day. The outcome was noted on visual analog score.

Data was analysed using the Statistical Package for Social Sciences (SPSS version 16). Median and interquartile were calculated for continuous variables, like age and pain score at 1-month and pain at six months. For the categorical variable, e.g. side of hernia frequencies were calculated. Mann-Whitney U-test was used to compare the pain between the two groups. A $p$-value of less than 0.05 was considered statistically significant. The outcome measure was groin pain at 1 and 6 months, compared in terms of intensity of pain. Median and Mann-Whitney U-test was applied.

\section{RESULTS}

One hundred and eighty consecutive indoor patients were enrolled on a convenient sampling basis with 90 in each group. Age range was 18 to 80 years. Median age of patients was 43 years and IQR $=35.5$ years $(n=180)$ with $53.33 \%$ (96) patients below the age of 45 years. Majority, i.e. $71.7 \%$ (129), had right sided hernia and $28.3 \%$ (51) had left sided hernia.
All patients who were included in the study completed their follow-up at 6 months in either group. Two patients from Group B had intractable pain and were managed by analgesics accordingly; but they went on to complete follow-up with nerve blocks. None of the patient in group A had any side effects causing any adjustments in the plan. Thus all 180 patients in the study of both the groups were available for follow-up.

At the end of six months, $77.77 \%$ (70) patients in the Group A and 60\% (54) in the Group B experienced no pain, $18.88 \%$ (17) patients in the Group $A$ and $25.55 \%$ (23) in the Group B experienced mild pain, $3.33 \%$ (3) patients in the Group A and $12.22 \%$ (11) in the Group B experienced moderate pain, $00 \%$ patients in the Group $A$ and $2.22 \%$ (2) in the Group B experienced severe pain.

Mann-Whitney $U$-test revealed significant difference in pain in the nerve-preservation group $(M d=6.00, I Q R=4$, $\mathrm{n}=90)$ and nerve-excision group ( $M d=3.50, I Q R=4, n=90$ ), $U=2301.00, z=-5.017, p<0.001$, indicating that prophylactic ilioinguinal neurectomy significantly reduces chronic inguinodynia following Lichenstein hernioplasty at one month. Mann-Whitney U-test also revealed significant difference in pain in the nerve-preservation group ( $M d=2.00, I Q R=1.00, n=90)$ and nerve-excision group ( $M d=0.00, I Q R=1.00, n=90), U=3001.00, z=-3.470$, $p=0.001$, indicating that prophylactic ilioinguinal neurectomy significantly reduces chronic inguinodynia following Lichenstein hernioplasty at six months, too.

\section{DISCUSSION}

Chronic inguinodynia incidence is widely underreported. Management of chronic inguinodynia has shifted in recent times from postoperative to prophylactic modalities because of the morbidity associated with the analgesics, repeat surgical procedure and the risk of recurrence of hernia. ${ }^{4}$ Tripple neurectomy remains the most widely advocated prophylactic as well as postoperative surgical treatment for chronic inguinodynia, but at the cost of numbness of inguinal area and other side effects.3,4,9-14 Few studies showed that ilioinguinal neurectomy alone can be employed for prevention of chronic inguinodynia with lesser degree of numbness and comparable pain incidence. The aim of this study was comparison of the efficacy of prophylactic ilioinguinal neurectomy against nerve preservation in alleviating chronic inguinodynia.

In a study by Zannoni and team, statistically significant decrease in incidence of chronic pain at 1-year occurred following long nerve resection compared to sectioning the nerve only. Moderate pain at 1 month; and intense pain at 1 week and 1 month was also decreased making nerve resection superior to simple section at preventing postoperative inguinodynia. 5 According to Basimbe et al., the incidence of postoperative chronic pain was significantly less in Lichtenstein hernioplasty with 
neurectomy group versus only Lichtenstein group at one month of operation. 6 At six months, however, there was no significant difference between both groups regarding groin pain at rest and coughing and sensorial changes in the two groups were similar. 6

In a study by Sumrat and coworkers, significantly loesser inguinodynia was observed in neurectomy group. ${ }^{3}$ However, in another randomised controlled trial, Joshi found lower incidence of chronic pain in the neurectomy group compared to the nerve preservation group. 8 In another study by Mattoo et al., the incidence of inguinodynia was significantly reduced in ilioinguinal neurectomy group than the preservation group. No significant differences were found regarding the incidence of postoperative sensory loss or paresthesias at the groin region postoperation. ${ }^{9}$ In a meta-analysis by Barazanchi and coworkers, groin pain occured less in neurectomy group after surgery in short and mid term, but has little effect on long term basis. ${ }^{12}$ Another meta analysis of RCTs reported significantly lower incidence of chronic groin pain in patients who had elective neurectomy, compared with controls. Furthermore, it advocated elective excision of the ilioinguinal nerve to be associated with additional morbidities of neurosensory disturbances or quality of life at 6-month. ${ }^{15}$ Based on Khoshmohabbat study, evaluation of postsurgical complications showed no significant difference between neurectomy and preservation group. This showed no increase in post-surgical complications after prophylactic excision of the ilioinguinal nerve. There was earlier return to work in the nerve-excised group. However, numbness was an inevitable complication, but pain relief is superior to numbness for patients.

Due to its presence in the operative field, ilioinguinalnerve identification and preservation or excision is pivitol. ${ }^{14,15}$ In addition, studies show that its excision involves lesser degree of morbidity as contralateral nerve fibers take over the area supplied by ilioinguinal nerve. ${ }^{12}$ In a randomised trial by Shah and coworkers, prophylactic neurectomy was found to be effective and safe option to prevent inguinodynia. ${ }^{16}$

This study was a short term follow-up study. On an average, nerve-preservation group had less score on assertion scale $(4.40 \pm 2.67)$, than nerve-excision group $(2.30 \pm 1.70)$. However, this difference was statistically significant indicating that prophylactic ilioinguinal neurectomy significantly reduces chronic inguinodynia following Lichenstein hernioplasty at six months. This supports the hypothesis that excision of ilioinguinal nerve significantly reduces chronic inguinodynia and it can be a routine in hernioplasty. The procedure is easy; but to establish the effectiveness of this procedure in various settings, it should further be worked upon. This study recommends that llioinguinal neurectomy should be considered as a routine surgical step during open mesh hernia repair.
There is no local study available so far in the literature, addressing chronic inguinodynia.

\section{CONCLUSION}

Prophylactic ilioinguinal neurectomy in Lichenstein hernioplasty significantly reduces chronic inguinodynia.

\section{REFERENCES}

1. Bjurstrom MF, Nicol AL, Amid PK, Chen DC. Pain control following inguinal herniorrhaphy: current perspectives. J Pain Res 2014; 7:277-90.

2. Khoshmohabat $H$, Panahi F, Alvandi AA, Mehrvarz S, Mohebi HA, Shams Koushki E. Effect of Ilioinguinal neurectomy on chronic pain following herniorrhaphy. Trauma Mon 2012: 17:323-8.

3. Sunkar S, Brashier DB, Bhagwat K, Sharma V, Angrish P. A clinical study of the incidence and disability caused by mesh neuralgia after inguinal mesh hernioplasty and the effects of prophylactic ilioinguinal neurectomy and its role in preventing chronic groin pain after inguinal hernioplasty. Int Surg J 2018; 5:1083-9.

4. Sivashanmugam T, Saraogi A, Smiles SR, Ravishankar MR. Ultrasound guided percutaneous electro-coagulation of ilioinguinal and iliohypogastric nerves for treatment of chronic groin pain. Indian J Anaesth 2013; 57:610-2.

5. Zannoni M, Luzietti E, Viani L, Nisi P, Caramatti C, Sianesi M. Wide resection of inguinal nerves versus simple section to prevent postoperative pain after prosthetic inguinal hernioplasty: Our experience. World J Surg 2014; 38:1037-43.

6. Basimbe F, Ongom PA, Kijjambu SC, Kituuka O. Postoperative pain in lichtenstein repair with Iliohypogastric neurectomy compared to standard lichtenstein repair, for inguinal hernias at Mulago Hospital, A Sub Saharan Africa Tertiary Centre. J Gen Pract 2013; 1:128.

7. Hakeem A, Shanmugam V. Current trends in the diagnosis and management of post hernioraphy chronic groin pain. World $J$ Gastrointest Surg 2011; 3:73-81.

8. Joshi A. Prophylactic ilioinguinal neurectomy: Can it prevent chronic groin pain after lichtenstein inguinal hernia repair? J Kathmandu Med Col 2016; 4:39-44.

9. Mattoo S, Paul V, Kachroo SL, Sharma R, Singh A, Hussain Z. Outcome of elective prophylactic ilioinguinal neurectomy in prevention of chronic groin pain after open inguinal hernia repair. J Evol Med Dent Sci 2016; 5:1992-7.

10. Acar F, Ozdemir M, Bayrakli F, Cirak B, Coskun E, Burchiel K. Management of medically intractable genitofemoral and ilioinguinal neuralgia. Turk Neurosurg 2013; 23:753-7.

11. Kline CM, Lucas CE, Ledgerwood AM. Directed neurectomy for treatment of chronic postsurgical neuropathic pain. $A m \mathrm{~J}$ Surg 2013; 205:246-8.

12. Barazanchi AW, Fagan PV, Smith BB, Hill AG. Routine neurectomy of inguinal nerves during open onlay mesh hernia repair: A meta-analysis of randomized trials. Ann Surg 2016; 264:64-72.

13. Bischoff JM, Enghuus C, Werner MU, Kehlet H. Long-term follow-up after mesh removal and selective neurectomy for persistent inguinal postherniorrhaphy pain. Hernia 2013; 17 : 339-46. 
14. Chen DC, Hiatt JR, Amid PK. Operative management of refractory neuropathic inguinodynia by a laparoscopic retroperitoneal approach. JAMA Surg 2013; 148:962-7.

15. Charalambous MP, Charalambous CP. Incidence of chronic groin pain following open mesh inguinal hernia repair, and effect of elective division of the ilioinguinal nerve: Meta- analysis of randomized controlled trials. Hernia 2018; 17:401-9.

16. Shah P, Aaudichya A, Juneja I, Vaishnani B, Rajyaguru A, Bhatt J. A comparative study between prophylactic ilioinguinal neurectomy versus nerve preservation in lichenstein tensionfree meshplasty for inguinal hernia repair. Indian $J$ Surg 2018; $1-7$

......... 